\title{
A NOTE ON TRANSFORMS OF SUBSPACES OF HILBERT SPACE
}

\author{
W. E. LONGSTAFF
}

\begin{abstract}
The transform of a family $\mathscr{F}$ of (closed linear) subspaces of a Hilbert space $H$ by an invertible (bounded, linear) operator $S$ on $H$ is the family of subspaces $\{S M: M \in \mathscr{F}\}$. It is shown that the set of projections $\left\{P_{S M}: M \in \mathscr{F}\right\}$ is closed in the uniform (respectively, strong, weak) operator topology if and only if the set of projections $\left\{P_{M}: M \in \mathscr{F}\right\}$ is uniformly (respectively, strongly, weakly) closed. This answers affirmatively a question raised by $\mathbf{K}$. J. Harrison.
\end{abstract}

1. Preliminaries. Let $B(H)$ denote the set of all (bounded linear) operators acting on the complex Hilbert space $H$. For any (closed linear) subspace $M$ of $H$ let $P_{M}$ denote the (orthogonal) projection with range $M$. If $S \in B(H)$ is invertible, let $S M$ be the subspace $\{S x: x \in M\}$. For any family $\mathscr{F}$ of subspaces of $H$ the transform of $\mathscr{F}$ by $S$ is the family of subspaces $\{S M$ : $M \in \mathscr{F}\}$. The family $\mathscr{F}$ is called uniformly (respectively, strongly, weakly) closed if the family of projections $\left\{P_{M}: M \in \mathscr{F}\right\}$ is closed in the uniform (respectively, strong, weak) operator topology. K. J. Harrison [1] asked if the transform of a strongly closed family of subspaces is strongly closed. It is shown that the answer is affirmative and remains so if 'strongly' is replaced by 'uniformly' or 'weakly'. The author thanks Dr. Harrison for his comments.

2. Results. The key to the proof of the above results is the following.

LEMma. If $S \in B(H)$ is invertible and $M \subseteq H$ is a subspace, the operator $T_{M}=1+S P_{M}\left(S^{*}-S^{-1}\right)$ is invertible with inverse $1-P_{S M}+P_{S M} S^{*-1} S^{-1}$. Moreover, $P_{S M}=T_{M}^{-1} S P_{M} S^{*}$ and

$$
\left\|T_{M}^{-1}\right\|<1+\left\|S^{*-1} S^{-1}\right\| \text {. }
$$

Proof. Let $x \in H$ be arbitrary and let $y=P_{S M} x$. Then

$$
x-y \in H \ominus S M=S^{*-1}(H \ominus M)
$$

so $S^{*}(x-y) \in H \ominus M$. Hence $P_{M} S^{*} x=P_{M} S^{*} y$. Also, $S^{-1} y \in M$ so

$$
y=S P_{M} S^{-1} y=-S P_{M}\left(S^{*}-S^{-1}\right) y+S P_{M} S^{*} x .
$$

Thus

$$
\left[1+S P_{M}\left(S^{*}-S^{-1}\right)\right] y=T_{M} P_{S M} x=S P_{M} S^{*} x
$$

Received by the editors July 13, 1978.

AMS (MOS) subject classifications (1970). Primary 46C05; Secondary 47B15. 
Since $x$ was arbitrary, $T_{M} P_{S M}=S P_{M} S^{*}$. It follows immediately that

$$
T_{M}\left[1-P_{S M}+P_{S M} S^{*-1} S^{-1}\right]=1 \text {. }
$$

Clearly $P_{S M} S P_{M}=S P_{M}$ and $P_{S M}=S P_{M} S^{-1} P_{S M}$. Taking adjoints of the latter gives $P_{S M}=P_{S M} S^{*-1} P_{M} S^{*}$. Hence

$$
P_{S M} S^{*-1} P_{M} S^{-1}=P_{S M} S^{*-1} S^{-1}
$$

Then

$$
\begin{aligned}
{\left[1-P_{S M}+P_{S M}\right.} & \left.S^{*-1} S^{-1}\right] T_{M} \\
= & 1+S P_{M} S^{*}-S P_{M} S^{-1}-P_{S M}-S P_{M} S^{*}+S P_{M} S^{-1} \\
& +P_{S M} S^{*-1} S^{-1}+P_{S M}-P_{S M} S^{*-1} S^{-1} \\
= & 1
\end{aligned}
$$

and the results follow.

THEOREM. If $\mathcal{F}$ is a family of subspaces of $H$ and $\mathcal{G}$ is the transform of $\mathcal{F}$ by the invertible operator $S \in B(H)$ then $\mathcal{G}$ is uniformly (respectively, strongly, weakly) closed if and only if $\mathcal{F}$ is uniformly (respectively, strongly, weakly) closed.

Proof. Since $\mathcal{F}$ is the transform of $\mathcal{G}$ by $S^{-1}$ we need only show that $\mathscr{F}$ uniformly (respectively, strongly, weakly) closed implies that $\mathcal{G}$ is uniformly (respectively, strongly, weakly) closed.

To establish the 'uniform' (respectively, 'strong') case, it suffices to show that if a net of projections $\left\{\boldsymbol{P}_{\boldsymbol{M}_{\alpha}}\right\}$ converges uniformly (respectively, strongly) to the (necessarily a projection) operator $P_{M}$, then the net $\left\{P_{S M_{\alpha}}\right\}$ converges uniformly (respectively, strongly) to the projection $P_{S M}$. By the lemma,

$$
\begin{gathered}
P_{S M_{\alpha}}=T_{M_{\alpha}}^{-1} S P_{M_{\alpha}} S^{*} \quad \text { where } T_{M_{\alpha}}=1+S P_{M_{\alpha}}\left(S^{*}-S^{-1}\right), \\
P_{S M}=T_{M}^{-1} S P_{M} S^{*} \quad \text { where } T_{M}=1+S P_{M}\left(S^{*}-S^{-1}\right),
\end{gathered}
$$

and $\left\|T_{M}^{-1}\right\|<1+\left\|S^{*-1} S^{-1}\right\|$ for every $\alpha$.

Let $P_{M_{\alpha}} \rightarrow P_{M}$ uniformly (respectively, strongly). Clearly $T_{M_{\alpha}} \rightarrow T_{M}$ uniformly (respectively, strongly). From the identity

$$
T_{M}^{-1}-T_{M_{\alpha}}^{-1}=T_{M_{\alpha}}^{-1}\left(T_{M_{\alpha}}-T_{M}\right) T_{M}^{-1}
$$

and the fact that $\left\{\left\|T_{M_{\alpha}}^{-1}\right\|\right\}$ is bounded it follows that $T_{M_{\alpha}}^{-1} \rightarrow T_{M}^{-1}$ uniformly (respectively, strongly). It is now a simple exercise to show that $P_{S M_{\alpha}} \rightarrow P_{S M}$ uniformly (respectively, strongly).

The 'weak' case is obtained from the 'strong' case and the compactness of the unit ball $B_{1}(H)$ of $B(H)$ in the weak operator topology as follows. If $\left\{P_{S M_{\alpha}}\right\}$ is a net converging weakly to the operator $E \in B(H)$ and $M_{\alpha} \in \mathcal{F}$ for every $\alpha$, then by weak compactness of $B_{1}(H)$ the net $\left\{P_{M_{\alpha}}\right\}$ has a subnet which converges weakly to an operator $F \in B_{1}(H)$. Since $\mathscr{F}$ is weakly closed, $F=P_{M}$ with $M \in \mathscr{F}$. This subnet then converges strongly to $\boldsymbol{P}_{M}$ and so $\boldsymbol{P}_{S M}$ 
is the strong limit of a subnet of $\left\{P_{S M_{a}}\right\}$. But this subnet converges weakly to $E$ so $E=P_{S M}$. Hence $\mathcal{G}$ is weakly closed.

The proof of the theorem is complete.

\section{REFERENCES}

1. K. J. Harrison, Transitive atomic lattices of subspaces, Indiana Univ. Math. J. 21 (1972), 621-642.

Departmant of Mathematics, University of Western Australia, Nedlands, Western Australia 6009, Australin 